Supporting Information

\title{
Long-Term Stable Recombination Layer for Tandem Polymer Solar Cells Using Self-Doped Conducting Polymers
}

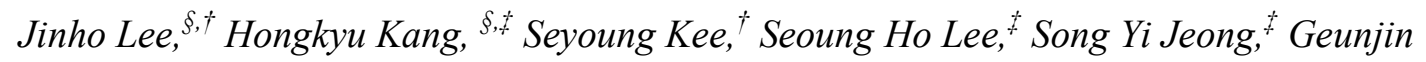

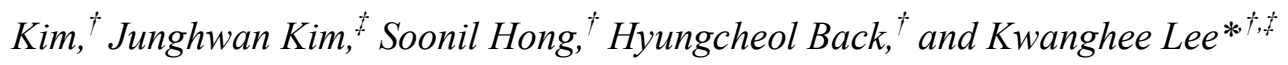

${ }^{\dagger}$ Department of Nanobio Materials and Electronics (DNE)

School of Materials Science and Engineering (SMSE)

Gwangju Institute of Science and Technology (GIST), Gwangju 500-712, Korea

* Research Institute for Solar and Sustainable Energies (RISE),

Gwangju Institute of Science and Technology (GIST), Gwangju 500-712, Korea

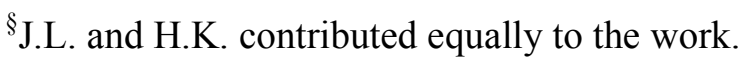

*E-mail: klee@gist.ac.kr (Kwanghee Lee). 
(a)

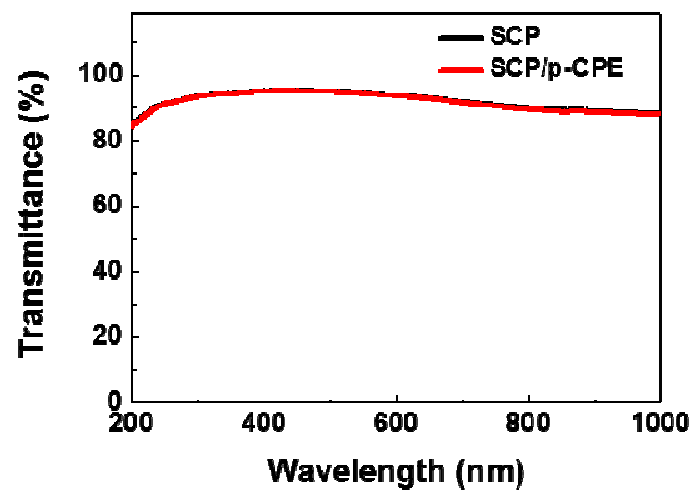

(b)

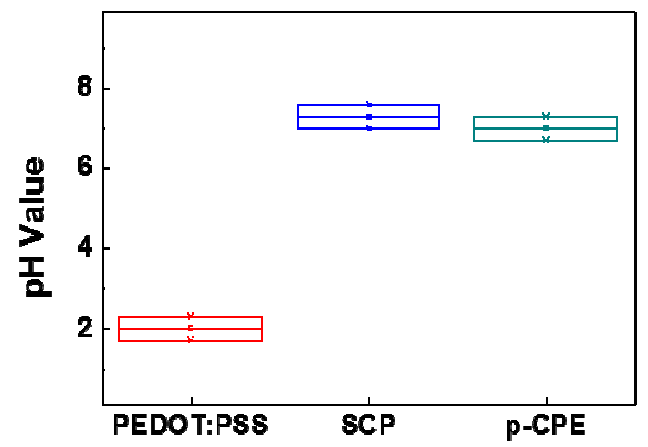

(c)

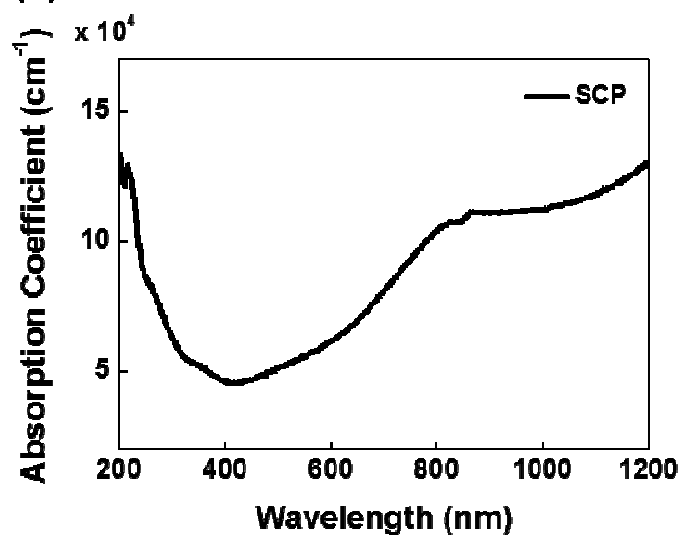

Figure S1. (a) Transmittance spectra of SCP and SCP/p-CPE. (b) pH values of PEDOT:PSS, SCP and p-CPE. (c) Absorption coefficient spectrum of the SCP film.

We confirmed that the optical and electrical properties of the SCP remain unchanged after the solution processing of p-CPE dissolved in alcohol solvent (e.g., methanol or 2methoxyethanol). Thus, we conclude that $\mathrm{pH}$-neutral P-CPE did not affect the intrinsic properties of the underlying SCP except for the surface energy level. 
(a)

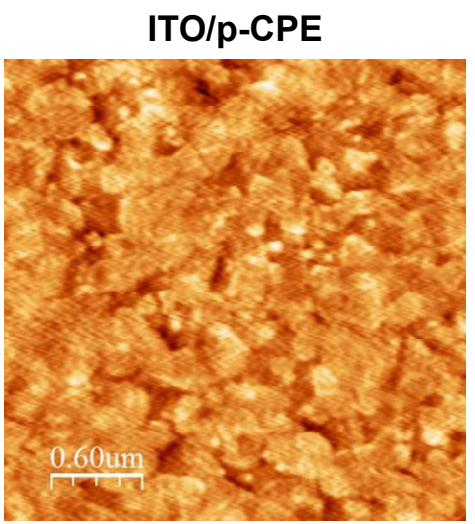

RMS : $1.53 \mathrm{~nm}$ (b)

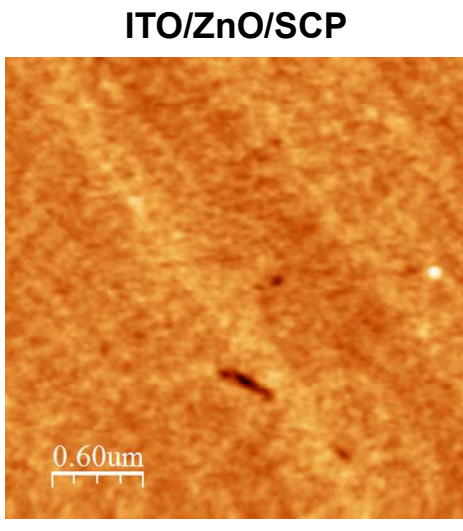

RMS : $0.56 \mathrm{~nm}$

Figure S2. Tapping-mode topographic AFM images of (a) ITO/p-CPE (b) ITO/ZnO/SCP. 
(a)

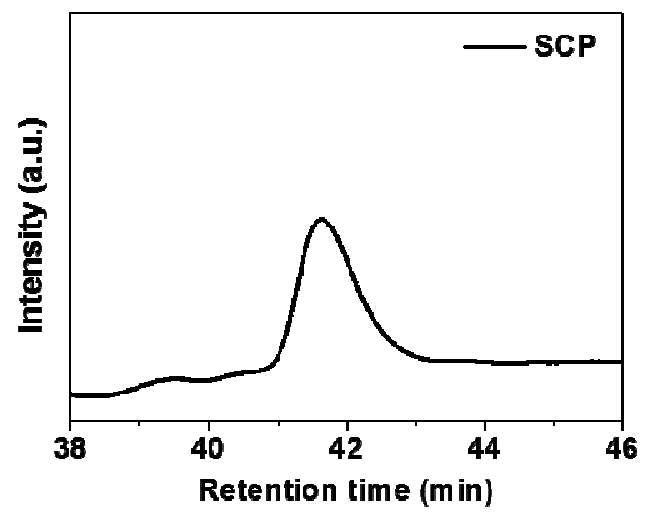

(b)

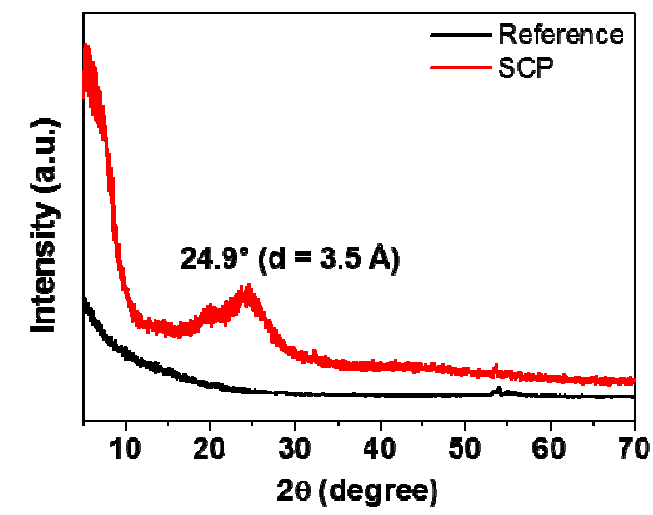

Figure S3. (a) GPC profile for the SCP and (b) XRD pattern of the SCP film. 


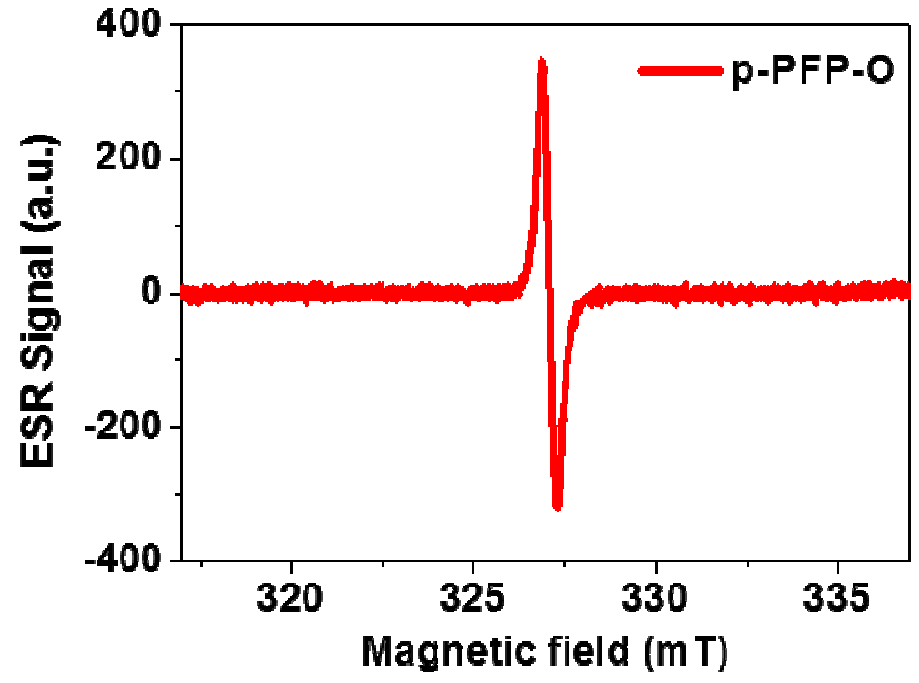

Figure S4. ESR spectrum of the p-CPE solution. 


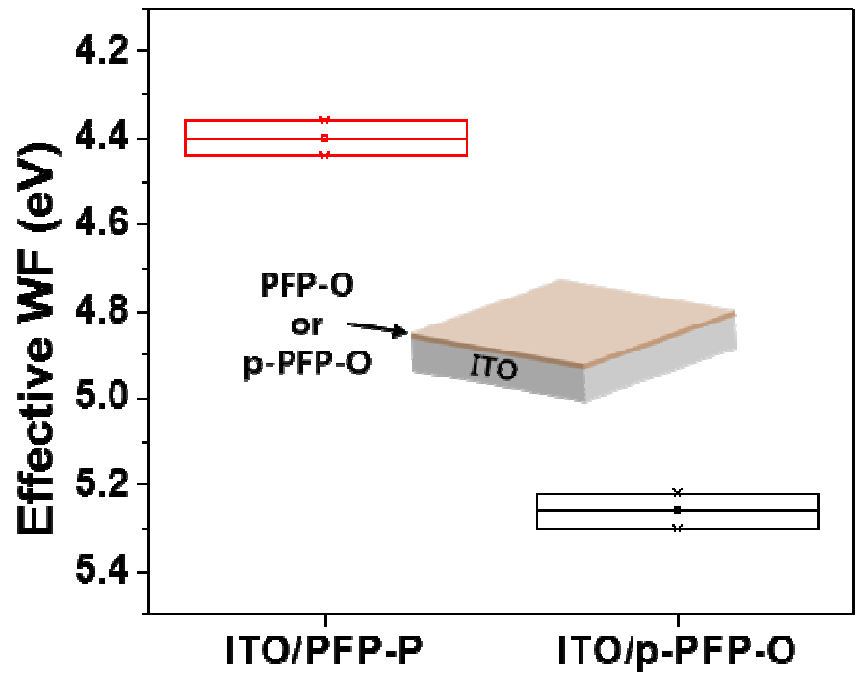

Figure S5. Effective WFs of ITO/PFP-O and ITO/p-PFP-O. 
(a)

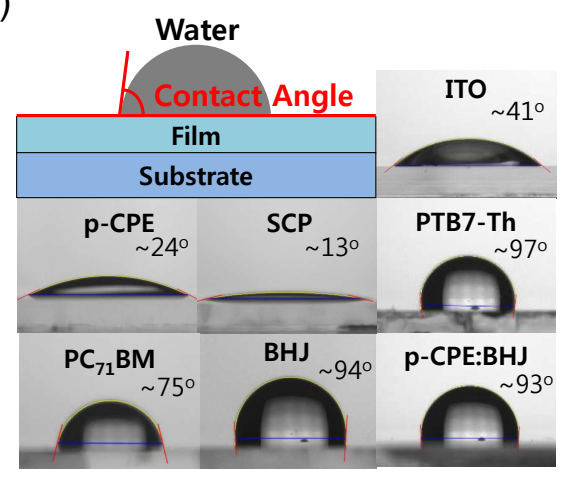

(b)

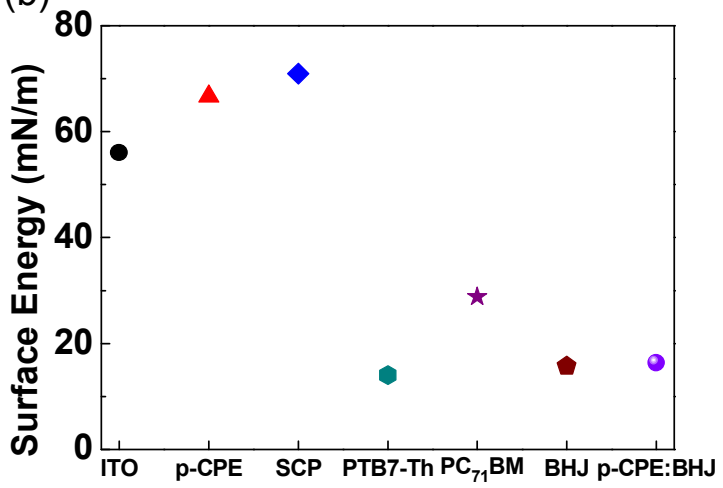

Figure S6. (a) Water contact angles and (b) corresponding surface energies of various materials. 


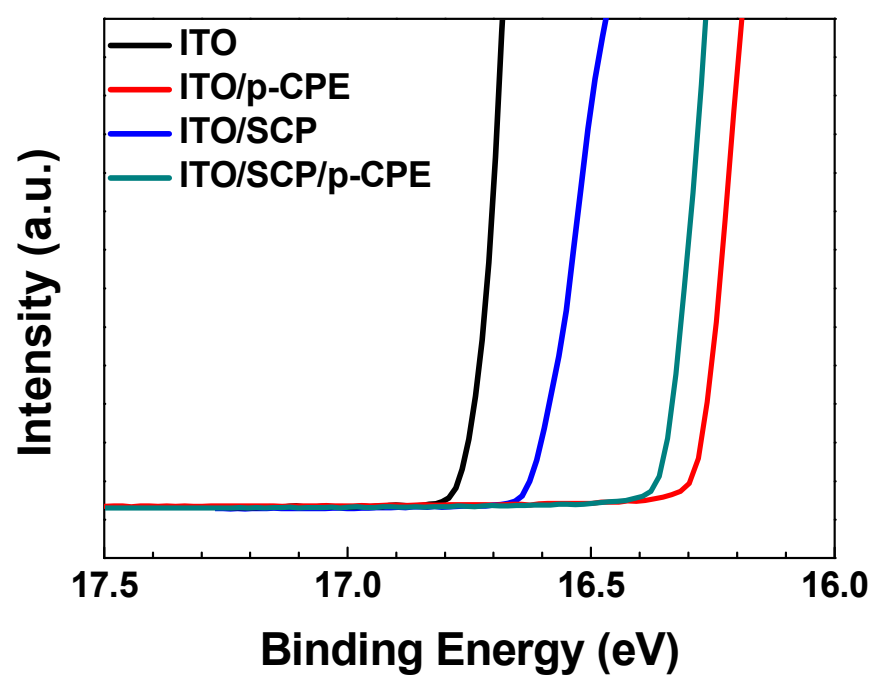

Figure S7. UPS spectra of ITO and SCP modified with p-CPE. 

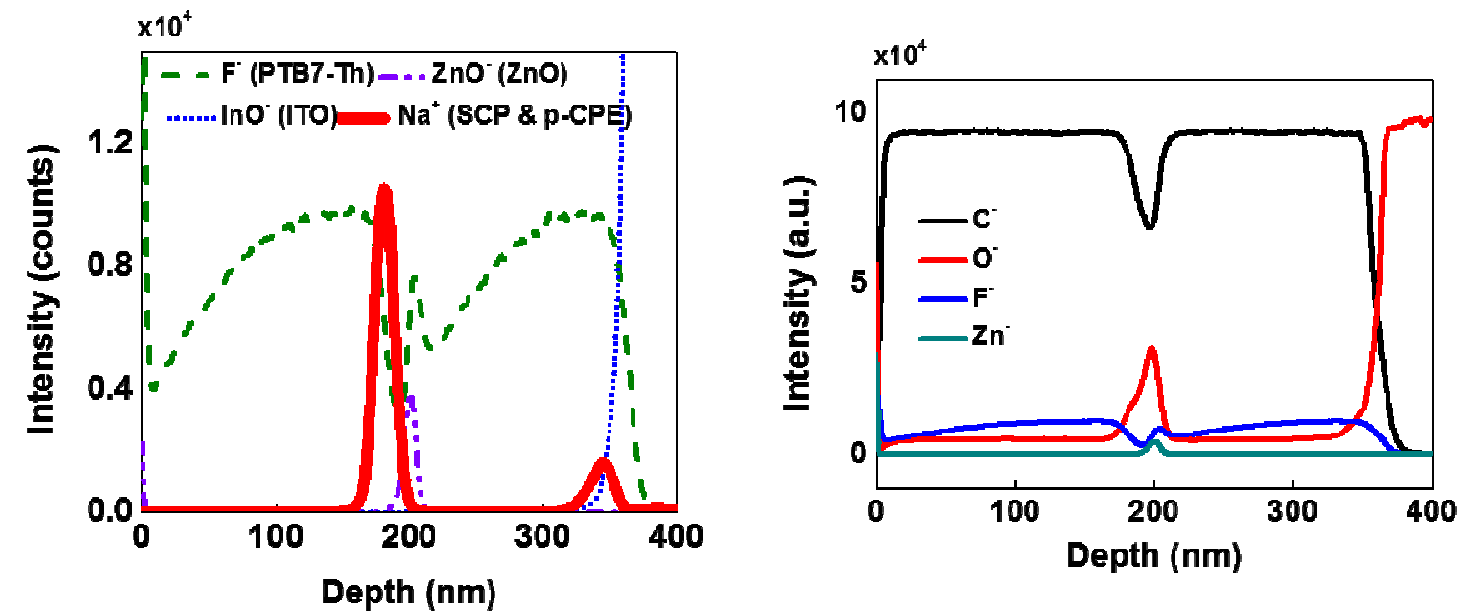

Figure S8. TOF-SIMS depth profiles for the tandem cell with p-CPE/BHJ layers. 
(a)

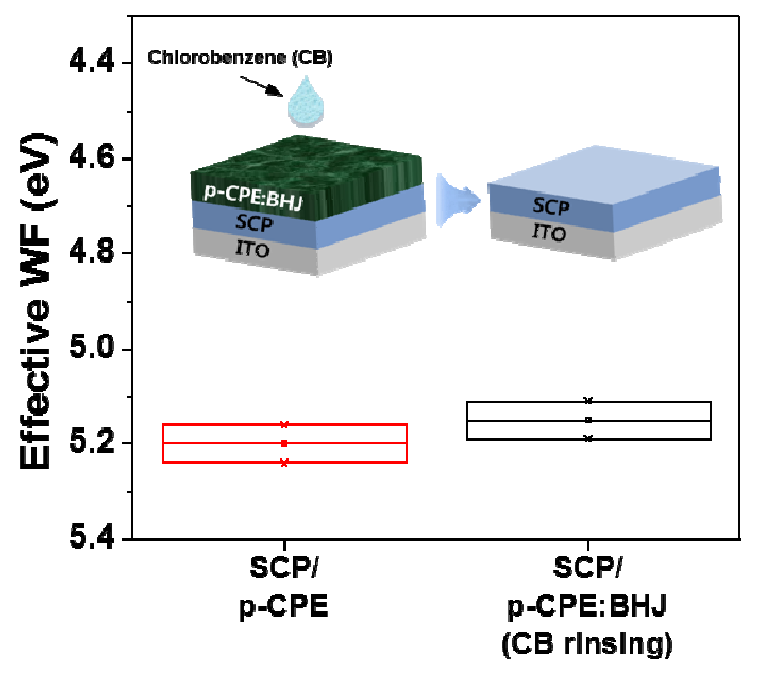

(b)

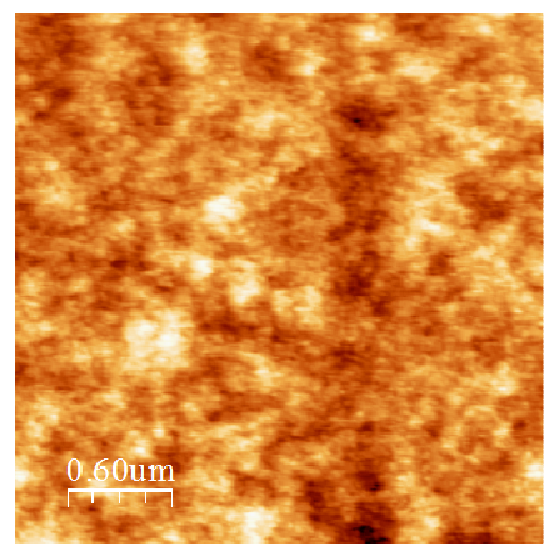

RMS : $0.93 \mathrm{~nm}$

Figure S9. (a) KP data and (b) tapping-mode topographic AFM image of ITO/SCP/p$\mathrm{CPE}$ :BHJ (CB rinsing). The inset in (a) describes the schematics for rinsing of $\mathrm{ITO} / \mathrm{SCP} / \mathrm{p}$ CPE:BHJ sample using CB. 
(a)

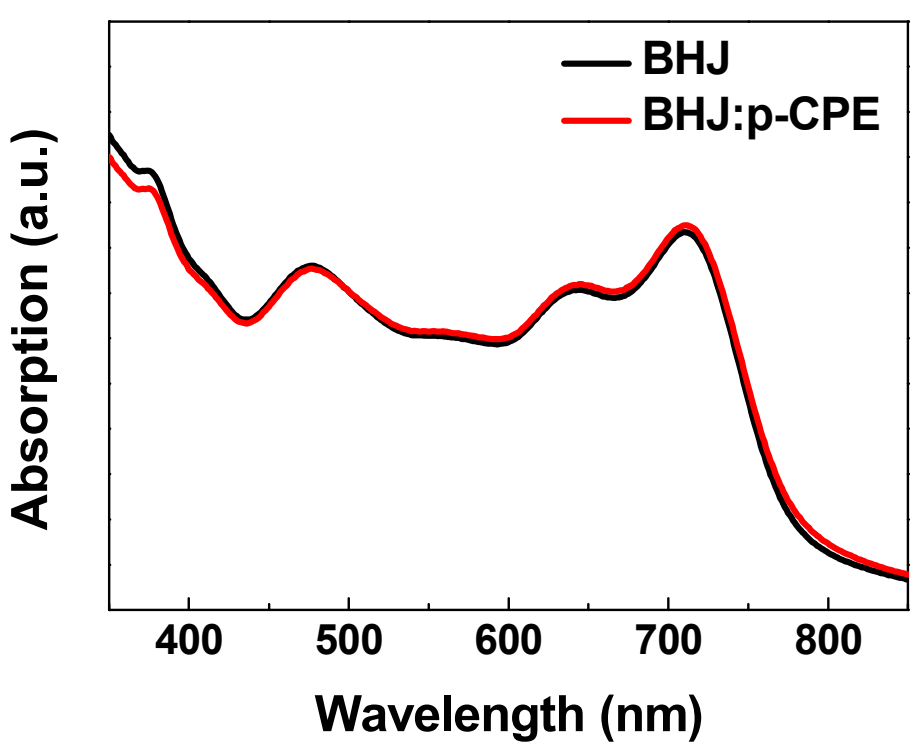

(b)

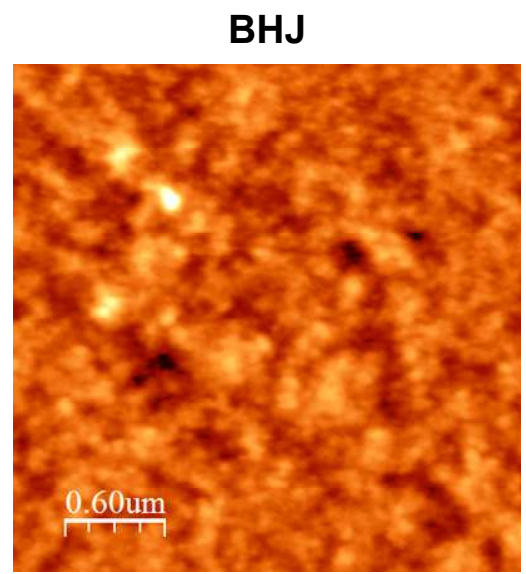

RMS : 1.99nm

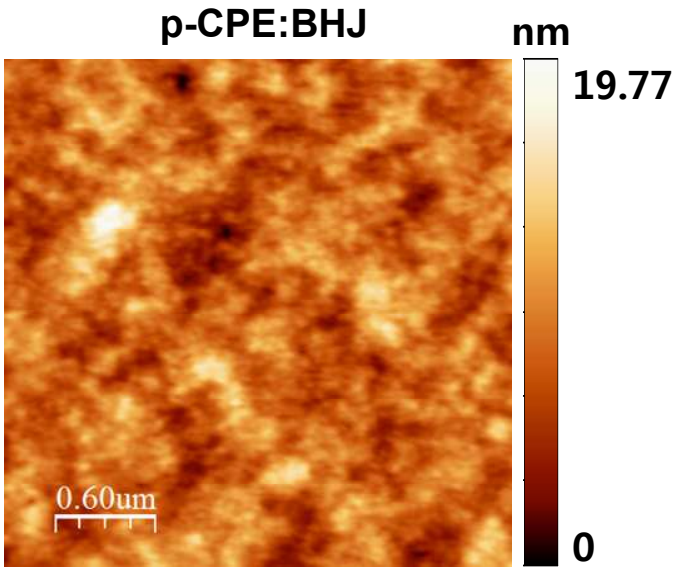

RMS : 2.04nm

Figure S10. (a) Absorption spectra of the BHJ and p-CPE:BHJ films. (b) Tapping-mode topographic AFM images of BHJ and p-CPE:BHJ. 
(a)
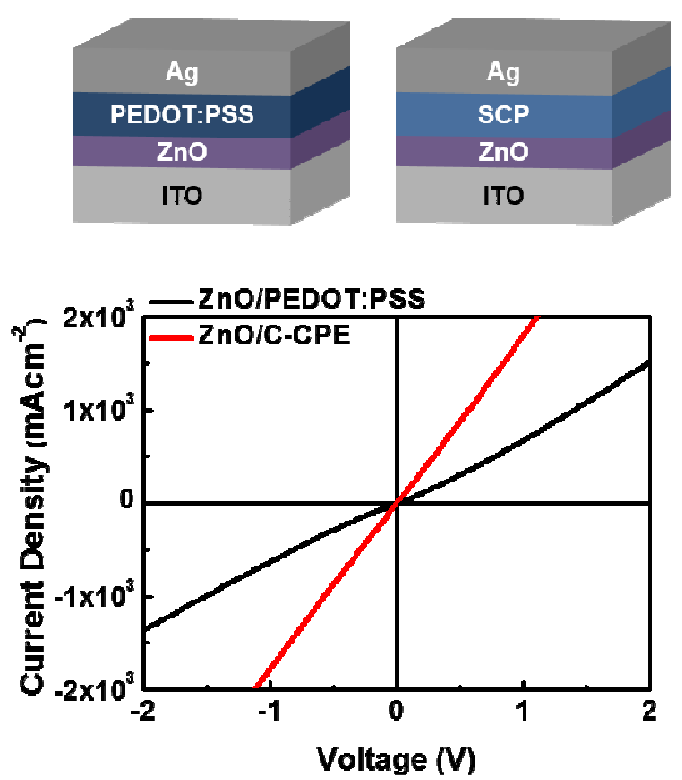

(b)
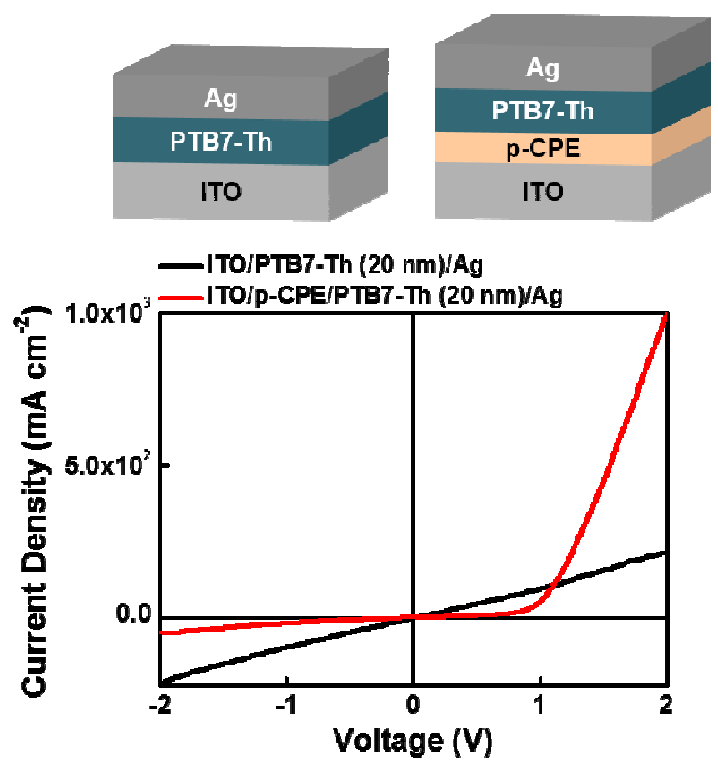

Figure S11. $J-V$ characteristics of (a) ITO/ZnO/PEDOT:PSS/Ag and ITO/ZnO/SCP/Ag, and (b) ITO/PTB7-Th/Ag and ITO/p-CPE/PTB7-Th/Ag. 
(a)

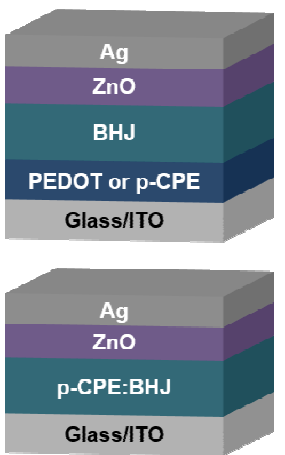

(b)

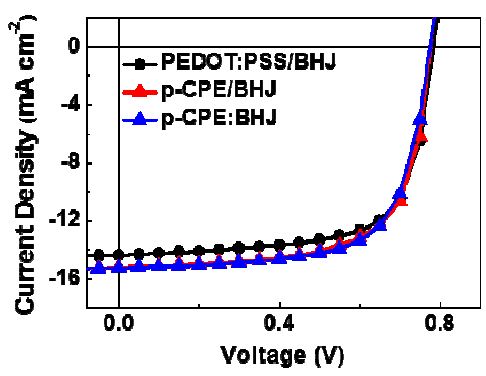

(c)

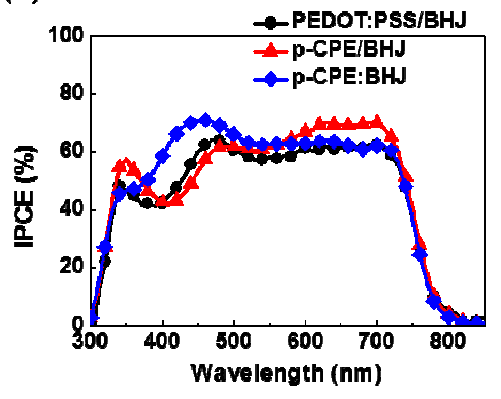

Figure S12. (a) Device structures, (b) $J-V$ characteristics and (c) IPCE spectra of front sub-cells based on the PEDOT:PSS/BHJ, p-CPE/BHJ, and p-CPE:BHJ.

Table S1. Performance parameters of front cells with different device configurations.

\begin{tabular}{|c|c|c|c|c|}
\hline Front Cell & $\begin{array}{l}V_{\text {oc }} \\
\text { (V) }\end{array}$ & $\begin{array}{c}\mathrm{J}_{\mathrm{sc}} \\
\left(\mathrm{mA} \mathrm{cm} \mathrm{cm}^{-2}\right)\end{array}$ & FF & $\begin{array}{c}\text { PCE } \\
(\%)\end{array}$ \\
\hline PEDOT:PSS/BHJ & 0.78 & 14.37 & 0.69 & 7.73 \\
\hline$p-C P E / B H J$ & 0.78 & 15.23 & 0.68 & 8.08 \\
\hline p-CPE:BHJ & 0.78 & 15.26 & 0.68 & 8.09 \\
\hline
\end{tabular}


(a)

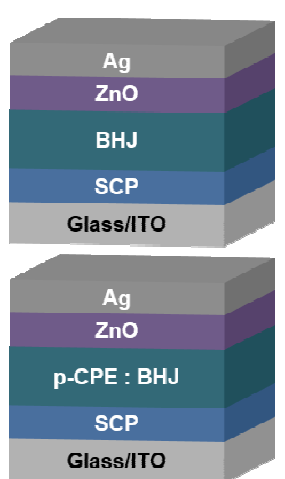

(b)

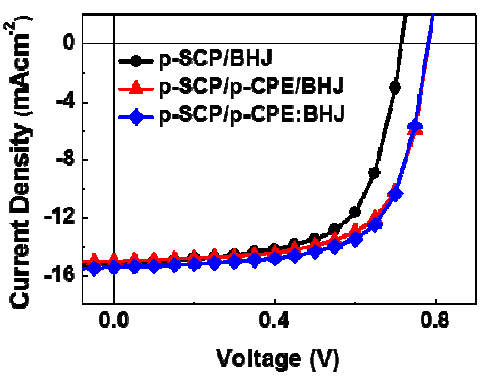

(c)

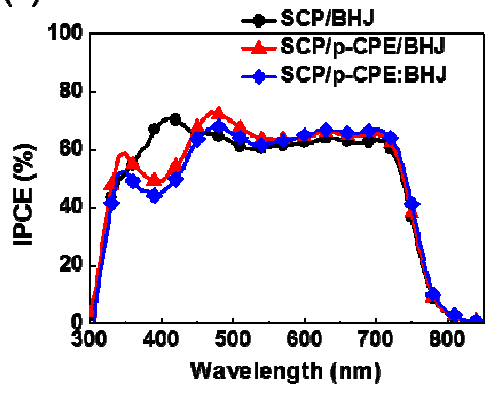

Figure S13. (a) Device structures, (b) $J-V$ characteristics and (c) IPCE spectra of back subcells based on the SCP/BHJ, SCP/p-CPE/BHJ, and SCP/p-CPE:BHJ.

Table S2. Performance parameters of back cells with different device configurations

\begin{tabular}{ccccc}
\hline Back Cell & $\begin{array}{c}\mathbf{V}_{\text {oc }} \\
(\mathbf{V})\end{array}$ & $\begin{array}{c}\mathbf{J}_{\text {sc }} \\
\left(\mathbf{m A ~ c m} \mathbf{~ c m}^{-2}\right)\end{array}$ & $\begin{array}{c}\text { FF } \\
\text { SCP/BHJ }\end{array}$ & $\begin{array}{c}\text { PCE } \\
\mathbf{( \% )}\end{array}$ \\
\hline SCP/p-CPE/BHJ & 0.72 & 15.20 & 0.65 & 7.11 \\
SCP/p-CPE:BHJ & 0.78 & 15.58 & 0.67 & 8.14 \\
\hline
\end{tabular}




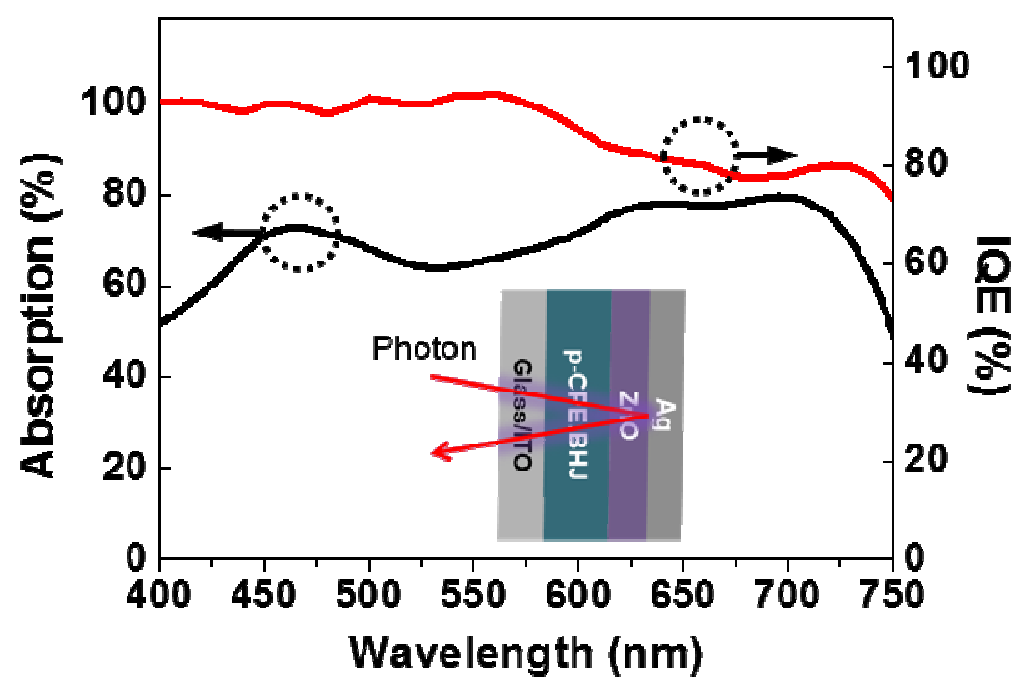

Figure S14. Absorption and internal quantum efficiency (IQE) spectra of the device with a structure of ITO/p-CPE:BHJ/ZnO/Ag. 